\title{
Antimony concentrations in nodal tissue can confirm sentinel node identity
}

\author{
Richard A Scolyer ${ }^{1,2}$, John F Thompson ${ }^{1,3}$, Ling-Xi L Li ${ }^{1}$, Alison Beavis ${ }^{4}$, Michael Dawson ${ }^{4}$, \\ Phillip Doble ${ }^{4}$, Robyne Soper ${ }^{2}$, Roger F Uren ${ }^{1,5}$, Jonathan R Stretch ${ }^{1,3}$, Ragwha Sharma ${ }^{1,2,6}$ \\ and Stanley W McCarthy ${ }^{1,2}$ \\ ${ }^{1}$ Sydney Melanoma Unit and Melanoma and Skin Cancer Research Institute, Royal Prince Alfred Hospital, \\ Camperdown, NSW, Australia; ${ }^{2}$ Department of Anatomical Pathology, Royal Prince Alfred Hospital, \\ Camperdown, NSW, Australia; ${ }^{3}$ Discipline of Surgery, Faculty of Medicine, University of Sydney, NSW, \\ Australia; ${ }^{4}$ Department of Chemistry, Materials and Forensic Science, University of Technology, Sydney, \\ NSW, Australia; ${ }^{5}$ Discipline of Medicine, Faculty of Medicine, University of Sydney, NSW, Australia and \\ ${ }^{6}$ Department of Anatomical Pathology, Institute of Clinical Pathology and Medical Research, Westmead \\ Hospital, Westmead, NSW, Australia
}

\begin{abstract}
The sentinel node biopsy procedure is a highly accurate method of staging patients with cutaneous melanoma and the tumor-harboring status of sentinel nodes is the most important prognostic factor. For the procedure to provide accurate prognostic information, however, it is essential that 'true' sentinel nodes are removed and examined thoroughly. A technique to confirm sentinel node identity may reduce the false-negative rate of the procedure. We have found that antimony (originating from the antimony sulfide colloid used for preoperative lymphoscintigraphy in our institution) can be measured in tissue sections of sentinel nodes using inductively coupled plasma mass spectrometry. The aims of this study were to determine whether antimony concentrations can be used to confirm that removed sentinel nodes are 'true' sentinel nodes and to differentiate sentinel nodes from nonsentinel nodes. In all, 24 patients who had both a tumor-positive sentinel node and a tumor-negative nonsentinel node removed from one regional node field during the same operation, were identified. Tissue sections $(50 \mu \mathrm{m})$ thick were cut from archival paraffin blocks of each of the sentinel nodes and nonsentinel nodes. Antimony concentrations in the tissue sections were measured using inductively coupled plasma mass spectrometry. The median and mean concentrations of antimony in parts per billion were 0.526 and 1.198, respectively (range $0.020-7.596$ ) in the sentinel nodes, and 0.043 and 0.123 (range $0-0.800$ ) in the nonsentinel nodes $(P=0.004)$. In four of the 24 pairs, both the presumed sentinel nodes and the nonsentinel nodes had very low antimony levels (less than $\mathbf{0 . 1 8}$ parts per billion), suggesting that nodes designated as sentinel nodes may not have been 'true' sentinel nodes. It is concluded that determination of antimony concentrations within sentinel nodes using the highly sensitive method of inductively coupled plasma mass spectrometry can confirm the identity of sentinel nodes and validate the sentinel node technique.

Modern Pathology (2004) 17, 1191-1197, advance online publication, 4 June 2004; doi:10.1038/modpathol.3800202
\end{abstract}

Keywords: antimony; mass spectrometry; melanoma; metastases; sentinel node

The technique of sentinel node biopsy has proved to be a highly accurate method of staging cutaneous melanoma. ${ }^{1-8}$ Furthermore, the presence or absence of metastases in the sentinel node is the most important prognostic factor for melanoma patients. ${ }^{9}$ For a sentinel node to provide accurate prognostic information, it is essential that 'true' sentinel nodes

Correspondence: Dr RA Scolyer, MBBS, Department of Anatomical Pathology, Royal Prince Alfred Hospital, Missenden Rd, Camperdown, NSW 2050, Australia.

E-mail: richard.scolyer@email.cs.nsw.gov.au

Received 9 March 2004; revised and accepted 23 April 2004; published online 4 June 2004 are removed and examined thoroughly. With long-term follow-up of patients with negative sentinel nodes, some develop a recurrence in the mapped and sampled nodal basin and these patients may be considered to have a false-negative result of their sentinel node biopsy procedure. A technique to confirm true sentinel node identity may reduce the false-negative rate of the procedure, which has been disappointingly high (up to $44 \%$ ) in several recently reported studies,,$^{5,10-19}$ or at least provide a retrospective explanation for a falsenegative result.

The sentinel node biopsy procedure is technically demanding and as it becomes more widely used, the 
training and skill of some of those performing it may be suboptimal. ${ }^{3,20-25}$ The procedure of sentinel node biopsy at the Sydney Melanoma Unit involves preoperative injection of a technetium-99m-labelled antimony trisulfide colloid for lymphoscintigraphy, and intraoperative injection of Patent Blue $\mathrm{V}$ dye (Guerbert, Aulney-Sous-Bois, France) at the primary melanoma site for visual identification of sentinel nodes. Because the blue dye does not survive the processing procedures necessary for histopathologic examination and because all the technetium$99 \mathrm{~m}$ has decayed by the time that sample is ready for analysis, these features cannot be used retrospectively to confirm that a 'true' sentinel node has been removed. Recently, Haigh et $a l^{26}$ reported that carbon particles can be used to confirm the identity of sentinel nodes in cutaneous melanoma. They found that after peritumoral injection of carbon prior to wide excision, all tumorpositive sentinel nodes contained carbon particles $(n=18)$ as well as $90 \%$ of tumor-negative sentinel nodes $(n=93)$. A potential problem with this technique, however, is that the presence of the carbon particles may obscure metastatic tumor cells within the sentinel node and result in a falsenegative pathologic diagnosis. Another disadvantage is that it cannot be used to assess archival tissue.

We have recently shown that antimony concentrations can be measured in tissue sections from archival paraffin blocks of sentinel nodes using inductively coupled plasma mass spectrometry. ${ }^{27}$ In the present study, we measured the antimony concentrations in 24 pairs of tumor-positive sentinel nodes and tumor-negative nonsentinel nodes, each pair removed from one regional node field during the same operation from individual patients, in an attempt to confirm that removed sentinel nodes were 'true' sentinel nodes, to differentiate sentinel nodes from nonsentinel nodes and to establish an antimony concentration threshold for optimally defining nodes as sentinel nodes or nonsentinel nodes. Furthermore, the intensity (brightness) of the images of each sentinel node on the preoperative lymphoscintigram was correlated with its antimony concentration to determine whether poor uptake of the colloid may have been the cause of the low antimony concentrations that were present in some sentinel nodes.

\section{Patients and methods}

Between March 1992 and June 2001, 1330 patients with a single primary cutaneous melanoma underwent sentinel node biopsy and were followed up at the Sydney Melanoma Unit. Patients were offered a sentinel node biopsy in the context of a randomized multicenter clinical trial after April 1994 if their tumors were $\geq 1 \mathrm{~mm}$ thick, and/or Clark level IV and there were no clinical signs of metastasis. A sentinel node could not be identified in 21 patients and was found to be positive in 176. In general, attempts were made to avoid removal of nonsentinel nodes whenever possible. However, a number of patients who had one or more sentinel nodes and one or more nonsentinel nodes removed from one regional node field during the same operation were identified. A subset of 24 patients who had one positive sentinel node and one negative nonsentinel node were selected, and formed the study group. The methods used to identify, remove and examine the sentinel nodes and nonsentinel nodes and to determine their antimony concentrations are detailed below.

\section{Lymphoscintigraphy}

After histopathologic confirmation of the diagnosis of cutaneous melanoma in the primary lesion by prior excision biopsy but before definitive wide excision, lymphoscintigraphy was performed. Details of the protocol used at the Sydney Melanoma Unit have been published previously. ${ }^{21,28}$ Briefly, four intradermal injections of technetium-99m antimony sulfide colloid (particle size $5-40 \mathrm{~nm}$ ), each $0.05-0.1 \mathrm{ml}$ in volume, were given around the primary melanoma site. Early and delayed imaging was then performed and the location of each sentinel node was marked with a skin tattoo spot and its depth beneath the skin surface was estimated and recorded.

\section{Operative Technique}

The sentinel nodes were biopsied within $24 \mathrm{~h}$ of the isotope-labelled colloid injection. Approximately $15 \mathrm{~min}$ prior to the operative procedure, a total of $1.0-2.0 \mathrm{ml}$ of blue dye (Patent Blue V, 2.5\%; Laboratoire Guerbert, Aulnay-Sous-Bois, France) was injected intradermally at four to six sites around the central part of the biopsy scar. A careful search for each sentinel node was conducted, guided by the lymphoscintigram and aided by blue dye visualization. A hand-held gamma probe was not used in the identification of sentinel nodes in the early Sydney Melanoma Unit experience of sentinel node biopsy. When it was used (from 1994 onwards), sentinel node identity was confirmed by a finding of radioactivity counts in the node that were at least three times the residual count in the node field. ${ }^{21}$ All nodes identified as sentinel nodes in the dynamic lymphoscintigraphy report were sought and removed if possible. The identity of the sentinel node was confirmed by the presence of blue staining in the node. Nonsentinel nodes were occasionally removed as well; these were defined as removed nodes lacking blue dye staining and with a radioactivity count less than three times the residual count in the node field. At the same operation, all patients were treated by wide excision of the 
primary site. Patient data and follow-up information used for this study were retrieved from a prospectively collected database.

\section{Histologic Assessment of Sentinel and Nonsentinel Nodes}

Sentinel nodes from all patients were cut along their longitudinal axes into $3 \mathrm{~mm}$ slices and entirely embedded in paraffin blocks following tissue processing. The presence of blue staining of the sentinel nodes was documented macroscopically on slicing the nodes. Four sequential $5-\mu \mathrm{m}$-thick tissue sections were cut from each block and stained with hematoxylin and eosin (H\&E) (on sections 1 and 4) and with immunohistochemical markers for S100 and HMB-45 (on sections 2 and 3). Removed nonsentinel nodes were also cut along their longitudinal axes into $3 \mathrm{~mm}$ slices and entirely embedded in paraffin blocks following tissue processing. Single $5-\mu$ m-thick tissue sections were cut from each block of the nonsentinel nodes and stained with $\mathrm{H} \& \mathrm{E}$. The sections of both the sentinel nodes and nonsentinel nodes were examined microscopically for the presence or absence of melanoma metastases, initially at a scanning magnification of $\times 100$, by histopathologists experienced in the assessment of melanoma and sentinel nodes. All of the sentinel nodes contained melanoma metastases and all nonsentinel nodes were negative for nodal metastases.

\section{Antimony Concentration Measurement}

The paraffin-embedded tissue blocks of the 24 pairs of sentinel and nonsentinel nodes were retrieved from the Anatomical Pathology Department of Royal Prince Alfred Hospital. Sections $50 \mu \mathrm{m}$ thick were cut from the tissue blocks of each sentinel node using a Jung Biocut 2035 rotary microtome (Leica Microsystems Pty Ltd, Australia). The tissue sections were digested using microwave generated heat as previously described, ${ }^{27}$ and the concentrations of antimony were measured using a Perkin-Elmer Sciex Elan 5100 inductively coupled plasma mass spectrometer with solution nebulization. ${ }^{27}$ All tissue samples were analyzed with no knowledge of whether they represented sentinel or nonsentinel nodes.

\section{Review of Lymphoscintigrams}

The lymphoscintigrams were available for review in 20 of the 24 cases. A single observer (RFU) graded the intensity of the images of each sentinel node as bright, medium, faint or absent.

\section{Statistical Analysis}

The paired $t$-test was employed to detect any significant difference between the antimony concentrations in the sentinel and nonsentinel nodes. The $P$-value for significance was set at a level of less than 0.05 .

\section{Results}

The antimony concentrations in the sections from the 24 pairs of sentinel and nonsentinel nodes are presented in Table 1 and Figure 1. The median and mean concentrations of antimony in the sections of the sentinel nodes were 0.526 and 1.198 parts per billion (range 0.020-7.596 parts per billion), whereas in the nonsentinel nodes the concentrations were 0.043 and 0.123 parts per billion (range 0-0.800 parts per billion). The sections of sentinel nodes had significantly greater antimony concentrations than those from the nonsentinel nodes $(P=0.004)$. The median concentration of antimony in the sections from all the lymph nodes (both

Table 1 Antimony concentration (in parts per billion) in $50-\mu \mathrm{m}-$ thick tissue sections of 24 pairs of sentinel and nonsentinel nodes removed from one regional node field during the same operation from individual patients. The intensity of the images of each sentinel node on review of the lymphoscintigraphy is also presented

\begin{tabular}{cccc}
\hline $\begin{array}{l}\text { Patient } \\
\text { no. }\end{array}$ & Antimony concentration (in ppb) & $\begin{array}{c}\text { Intensity of } S N \\
\text { on } L S G\end{array}$ \\
\cline { 2 - 3 } & $S N$ & Non-SN & \\
\hline 1 & 0.409 & 0.054 & ++ \\
2 & 0.327 & 0.104 & +++ \\
3 & 1.005 & 0.047 & +++ \\
4 & 0.203 & 0.038 & NS \\
5 & 0.322 & 0.129 & +++ \\
$6^{\mathrm{a}}$ & 0.020 & 0.036 & $\mathrm{UA}$ \\
$7^{\mathrm{a}}$ & 0.112 & 0.014 & + \\
$8^{\mathrm{a}}$ & 0.023 & 0.018 & $\mathrm{NS}$ \\
9 & 0.266 & 0.038 & $\mathrm{UA}$ \\
10 & 1.389 & 0 & +++ \\
11 & 0.277 & 0.040 & +++ \\
12 & 3.029 & 0.034 & $\mathrm{UA}$ \\
$13^{\mathrm{a}}$ & 0.036 & 0.059 & +++ \\
14 & 0.591 & 0.033 & +++ \\
15 & 0.915 & 0.228 & +++ \\
16 & 2.699 & 0.327 & +++ \\
17 & 2.160 & 0.023 & +++ \\
18 & 7.596 & 0.005 & +++ \\
19 & 3.257 & 0.653 & +++ \\
20 & 1.209 & 0.800 & +++ \\
21 & 0.461 & 0.062 & +++ \\
22 & 0.415 & 0.003 & +++ \\
23 & 1.324 & 0.045 & +++ \\
24 & 0.711 & 0.160 &
\end{tabular}

No. =number; ppb = parts per billion; $\mathrm{SN}=$ sentinel node; $\mathrm{LSG}=$ lymphoscintigram; Non-SN $=$ nonsentinel node; $\quad+++=$ bright; $++=$ medium; +=faint; NS = sentinel node assessed not seen on lymphoscintigraphy; UA = lymphoscintigraphy unavailable for review. ${ }^{a}$ Both sentinel and nonsentinel nodes had very low antimony level (less than 0.18 parts per billion). 


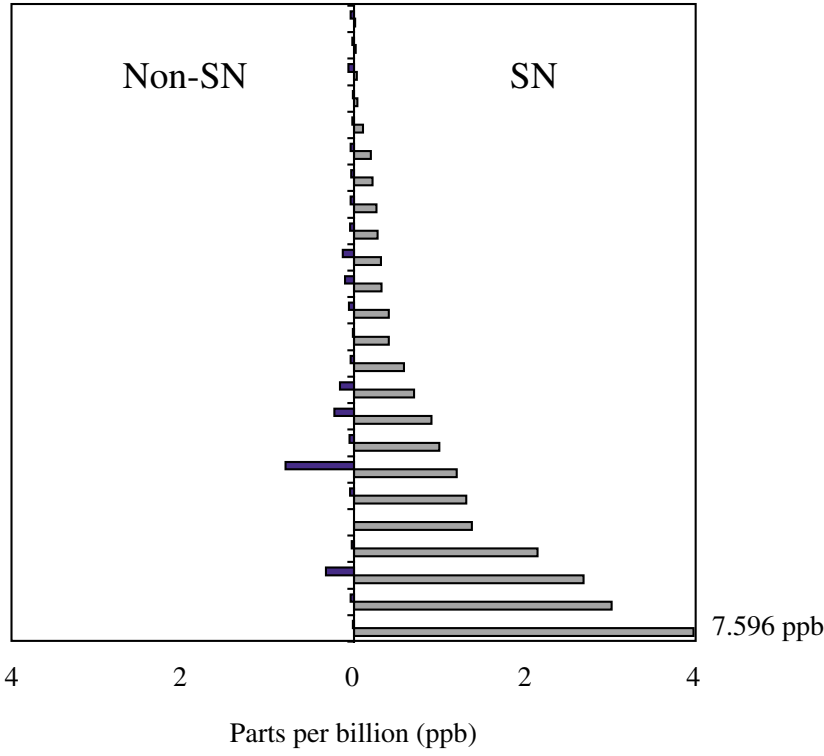

Figure 1 Antimony concentrations (in parts per billion) in $50-\mu \mathrm{m}-$ thick tissue sections of 24 pairs of sentinel nodes (SNs) and nonsentinel nodes (non-SNs) removed from one regional node field during the same operation from individual patients.

sentinel and nonsentinel nodes) was 0.18 parts per billion. In four of the 24 pairs, both sentinel and nonsentinel nodes had very low antimony levels (less than 0.18 parts per billion). If the median concentration of antimony ( 0.18 parts per billion) was selected to differentiate sentinel from nonsentinel nodes, 20 of 24 sentinel and 20 of 24 nonsentinel nodes were correctly identified, giving a detection sensitivity of $83 \%$ and a specificity of $83 \%$.

The results of the grading of the intensity of the images of each sentinel node on lymphoscintigraphy are also presented in Table 1 .

\section{Discussion}

The assessment of sentinel nodes requires a team approach involving surgeons, nuclear medicine physicians and pathologists., ${ }^{3,8,20-25}$ Technical failures may occur as a result of errors in lymphatic mapping and sentinel lymphadenectomy or because of a deficiency in the process of histopathologic evaluation. With long-term follow-up of patients with negative sentinel nodes there is a small but definite incidence of recurrence in the mapped and sampled nodal basin. ${ }^{5,10-19}$ These patients may be considered to have a false-negative result of their sentinel node biopsy procedure. Previous reports have suggested that the majority of patients who recur in the relevant node field will, in fact, have identifiable metastases in the biopsied sentinel node if a more comprehensive histopathologic evaluation of the node is undertaken. ${ }^{10,14}$ However, a review of the results of sentinel node biopsy procedures at the Sydney Melanoma Unit has suggested that an incorrect histopathologic diagnosis has been responsible for only a minority of regional failures in Sydney Melanoma Unit patients. ${ }^{15}$ One possible reason for this is that all sentinel node specimens have been assessed with multiple sections and immunohistochemical stains since the introduction of the technique at our institution.

\section{Failure to Remove 'True' Sentinel Nodes as a Cause of False-Negative Sentinel Node Biopsy Procedures}

In some patients, recurrence in a node field from which a sentinel node has previously been removed, and reported to be negative, occurs at the same time or subsequent to recurrence at distant sites. In such patients, the node field recurrence may represent a manifestation of systemic disease. However, in other patients the occurrence of false-negative sentinel nodes can be attributed to errors in lymphatic mapping by the nuclear medicine physician, errors by the surgeon at the time of sentinel lymphadenectomy or errors by the pathologist who undertakes histopathologic examination. A technique to determine whether the sentinel node removed is a 'true' sentinel node has the potential to identify surgical causes of false-negative sentinel node biopsies, that is, those in which the failure may be attributable to an error by the surgeon. The only previously reported method to retrospectively confirm that removed sentinel nodes are 'true' sentinel nodes is by the visual identification of large, dense carbon particles in histologic tissue sections of sentinel nodes as described by Haigh et $a l^{26}$ from the John Wayne Cancer Institute. A potential problem with their technique is that it may cause a false-negative result if the carbon particles obscure metastatic tumor cells. And, of course, the technique can only be used if carbon particles have been injected, so that retrospective assessment of the sentinel nodes of patients who underwent routine mapping with blue dye and a radio-labelled colloid cannot be performed.

We have recently found that measuring antimony concentrations by inductively coupled plasma mass spectrometry is a highly sensitive and specific method of differentiating sentinel nodes from lymph nodes and other tissues removed from nonmelanoma patients. ${ }^{27}$ This technique has the great advantage over the carbon particle technique described by Haigh et al in that it can be employed within existing pathologic practices used to examine sentinel nodes, and can be also applied to archival tissue. In the current study, we have shown that the measurement of antimony concentrations using inductively coupled plasma mass spectrometry on paired sentinel node and nonsentinel nodes, removed from the same regional lymph node field of individual patients during the same operative procedure, is highly accurate in differentiating sentinel nodes from nonsentinel nodes. This 
technique can thus be used to confirm retrospectively that removed sentinel nodes are in fact 'true' sentinel nodes.

\section{Localization of Colloid in Sentinel Nodes}

It is not known whether the colloid used for lymphoscintigraphy (such as antimony) is localized within a particular region of the lymph node, although it seems likely that the highest concentrations will be around the site of entry of afferent lymphatic channels. If the antimony is localized to a particular region of the lymph node, factors such as the plane of slicing of the sentinel node before it is processed for microscopic evaluation may affect the concentration of antimony detected in the sections cut from archival paraffin blocks. This could explain why four of the sentinel nodes analyzed in this study had low antimony levels. Interestingly, in the study by Haigh et $a l^{26}$ in which the presence of carbon particles were detected within sentinel nodes, it was noted that in most cases, the carbon particles were most prominent around the entry point of the afferent lymphatic. Potentially, antimony may also be localized in this region of the sentinel node. Further evidence in support of this hypothesis is a recent study of sentinel nodes from breast cancer patients, in which most metastatic tumor cell deposits were detected in that half of the node into which the afferent lymphatic channel entered. ${ }^{29}$ In a previous study, we attempted to measure the concentrations of antimony in different regions of sentinel nodes by assessing the antimony concentrations in tissue sections cut at various planes through the sentinel node. ${ }^{27}$ We found that there was some variation in the concentrations, suggesting that the concentration of antimony does indeed vary within individual nodes but perhaps less than expected, given the findings of the study using carbon pigment. Differences in the size of the particles and their transportation within the node are potential explanations of this apparent discrepancy. Alternatively, of course, it is possible that the nodes with low antimony concentrations were not true sentinel nodes but second-tier nodes.

\section{Low Antimony Concentrations in Sentinel Nodes-Possible Explanations}

In this study, we found that antimony concentrations were low in both the sentinel and the nonsentinel node in four of the patients. Possible explanations include the possibility that both nodes were nonsentinel nodes, poor migration of antimony from the injection site to the sentinel node, the presence of tumor in the sentinel node itself impeding subsequent uptake of labelled colloid, regional variations of antimony concentrations within the sentinel node or technical errors.
As each of the four sentinel nodes but none of the nonsentinel nodes contained metastatic tumor, it is probable that each sentinel node was a 'true' sentinel node. This is supported by the operative finding that each of the putative sentinel nodes was blue stained following Patent Blue injection at the primary melanoma site.

Poor migration of antimony from its site of injection in the skin to the sentinel node may occur for a number of reasons, including low ambient temperature, however, we make strenuous efforts to avoid this when a lymphoscintigram is performed. Poor migration may also occur because of a reduction in cutaneous tissue elasticity at the injection site. The reduction in tissue elasticity may result in only a minimal increase in interstitial tissue hydrostatic pressure following injection of the antimony-containing colloid and therefore poor flow of the colloid in lymphatic channels to the sentinel node. We have observed this clinically, particularly in elderly patients, in whom obviously reduced tissue elasticity is associated with poor visualization of sentinel nodes on their lymphoscintigraphy. The intensity of the images of the sentinel nodes on review of the lymphoscintigraphy gives some support to this hypothesis. In 17 of the 20 cases in which the lymphoscintigraphy was available for review, the sentinel nodes were graded as bright (16 cases) or medium (one case) and the antimony concentrations in the corresponding sentinel nodes were all much greater than in the paired nonsentinel nodes. In one case, the sentinel node was graded as faint on lymphoscintigraphy and the antimony concentration was low $(<0.18$ parts per billion) in both the sentinel and nonsentinel nodes. In two other cases, the examined sentinel node was not identified on the preoperative lymphoscintigraphy, and the antimony concentrations in the sentinel nodes were below or around 0.18 parts per billion. This suggests that in the four cases with low antimony concentrations in both the sentinel and nonsentinel nodes, the sentinel nodes had very poor uptake of the antimony-containing colloid, and indicates that this is the likely reason for their low antimony concentrations.

Although the concentration of antimony in individual samples may correlate with factors such as the size of the lymph node, the volume of nodal tissue in the sentinel node tissue sections analyzed and the number of sentinel nodes, we do not believe that these factors are likely to have had a major impact on our results or can explain the low antimony levels in these four pairs of samples. Because the differences between the concentrations of antimony in the sentinel and the nonsentinel nodes in most samples were so large (in many cases the concentrations were more than 100 times greater in the sentinel node) it appears unlikely that these factors would have affected the results to such a degree. 
What Antimony Concentration Differentiates Sentinel from Nonsentinel Nodes?

If the median antimony concentration of all 48 sentinel and nonsentinel nodes (0.18 parts per billion) is used to differentiate between sentinel nodes and nonsentinel nodes, a detection sensitivity and specificity of $83 \%$ ( 20 of 24 cases) is obtained. If the four cases with low antimony concentrations in both the sentinel and nonsentinel node are excluded for the current analysis, then a concentration threshold of 0.18 parts per billion would correctly identify 20 of 20 sentinel and 16 of 20 nonsentinel nodes. Further study is needed with larger number of cases to validate and optimize the antimony concentration threshold for differentiating sentinel from nonsentinel nodes.

The availability and cost of the equipment required for inductively coupled plasma mass spectrometry may limit the general applicability of determining antimony concentrations to confirm sentinel node identity in a prospective manner outside a university setting. However, the technique offers the ability to analyze all cases retrospectively, unlike the carbon particle method, which can only be used when carbon particles were injected at the primary melanoma site prior to sentinel node biopsy. We have used this method to determine that some cases of a false-negative sentinel node biopsy were caused by failure of sentinel lymphadenectomy, that is, the removed node designated as 'sentinel' was not in fact a true sentinel node. $^{30}$ Surgical error has not been previously highlighted as a cause of false-negative sentinel node biopsy.

In more recent studies at the Sydney Melanoma Unit, we have been attempting to analyze antimony concentrations in fine-needle aspirates obtained from lymph nodes, and have found that the technique is sufficiently sensitive to permit differentiation between sentinel and nonsentinel nodes by analysis of a fine-needle aspirate. Antimony concentrations of appropriately prepared samples can be determined by inductively coupled plasma mass spectrometry within a few minutes so that this technique has the potential to provide a rapid and reliable method of confirming the identity of true sentinel nodes intraoperatively, and could thereby reduce the false-negative rate for the sentinel node biopsy technique. If the presence or absence of metastatic melanoma in a node could also be determined from a fine-needle aspirate, this validation technique would be very valuable in confirming that the correct node had been sampled. Preliminary studies that we have undertaken using magnetic resonance spectroscopy to examine fine-needle aspirates ${ }^{31}$ suggest that it may indeed be possible to use this minimally invasive technique to assess sentinel nodes quickly and accurately.

\section{Conclusions}

Determination of antimony concentrations within lymph nodes using the highly sensitive technique of inductively coupled plasma mass spectrometry can confirm the identity of sentinel nodes and validate the sentinel node technique. Potentially inductively coupled plasma mass spectrometry could be used to confirm the identity of sentinel nodes intraoperatively.

\section{Acknowledgements}

The financial support of the University of Sydney Cancer Research Fund, the Royal College of Pathologists of Australasia, the Melanoma and Skin Cancer Research Institute and the Melanoma Foundation of the University of Sydney is gratefully acknowledged.

\section{References}

1 Morton DL, Wen DR, Wong JH, et al. Technical details of intraoperative lymphatic mapping for early stage melanoma. Arch Surg 1992;127:392-399.

2 Thompson JF, McCarthy WH, Bosch CM, et al. Sentinel lymph node status as an indicator of the presence of metastatic melanoma in regional lymph nodes. Melanoma Res 1995;5:255-260.

3 Morton DL, Thompson JF, Essner R, et al. Validation of the accuracy of intraoperative lymphatic mapping and sentinel lymphadenectomy for early-stage melanoma: a multicenter trial. Multicenter Selective Lymphadenectomy Trial Group. Ann Surg 1999; 230:453-463.

4 Gershenwald JE, Thompson W, Mansfield PF, et al. Multi-institutional melanoma lymphatic mapping experience: the prognostic value of sentinel lymph node status in 612 stage I or II melanoma patients. J Clin Oncol 1999;17:976-983.

5 Jansen L, Nieweg OE, Peterse JL, et al. Reliability of sentinel lymph node biopsy for staging melanoma. Br J Surg 2000;87:484-489.

6 Cascinelli N, Belli F, Santinami M, et al. Sentinel lymph node biopsy in cutaneous melanoma: the WHO Melanoma Program experience. Ann Surg Oncol 2000;7:469-474.

7 Clary BM, Brady MS, Lewis JJ, et al. Sentinel lymph node biopsy in the management of patients with primary cutaneous melanoma: review of a large single-institutional experience with an emphasis on recurrence. Ann Surg 2001;233:250-258.

8 Reintgen D, Ross MI, Essner R. Sentinel lymph node biopsy: results to date. In: Thompson JF, Morton DL, Kroon BB (eds). Textbook of Melanoma, 1st edn. Martin Dunitz: London, 2004, pp 365-372.

9 Balch CM, Soong SJ, Gershenwald JE, et al. Prognostic factors analysis of 17,600 melanoma patients: validation of the American Joint Committee on Cancer melanoma staging system. J Clin Oncol 2001;19: 3622-3634.

10 Gershenwald JE, Colome MI, Lee JE, et al. Patterns of recurrence following a negative sentinel lymph node 
biopsy in 243 patients with stage I or II melanoma. J Clin Oncol 1998;16:2253-2260.

11 Gadd MA, Cosimi AB, Yu J, et al. Outcome of patients with melanoma and histologically negative sentinel lymph nodes. Arch Surg 1999;134:381-387.

12 Statius Muller MG, Borgstein PJ, Pijpers R, et al. Reliability of the sentinel node procedure in melanoma patients: analysis of failures after long-term followup. Ann Surg Oncol 2000;7:461-468.

13 Harlow SP, Krag DN, Ashikaga T, et al. Gamma probe guided biopsy of the sentinel node in malignant melanoma: a multicentre study. Melanoma Res 2001;11:45-55.

14 Clary BM, Mann B, Brady MS, et al. Early recurrence after lymphatic mapping and sentinel node biopsy in patients with primary extremity melanoma: a comparison with elective lymph node dissection. Ann Surg Oncol 2001;8:328-337.

15 Li L-XL, Scolyer RA, Ka VSK, et al. Pathologic review of negative sentinel lymph nodes in melanoma patients with regional recurrence: a clinicopathologic study of 1152 patients undergoing sentinel lymph node biopsy. Am J Surg Pathol 2003;27: 1197-1202.

16 Wagner JD, Ranieri J, Evdokimow DZ, et al. Patterns of initial recurrence and prognosis after sentinel lymph node biopsy and selective lymphadenectomy for melanoma. Plast Reconstr Surg 2003;112:486-497.

17 Estourgie SH, Nieweg OE, Valdes Olmos RA, et al. Review and evaluation of sentinel node procedures in 250 melanoma patients with a median follow-up of 6 years. Ann Surg Oncol 2003;10:681-688.

18 Nowecki ZI, Rutkowski P, Nasierowska-Guttmejer A, et al. Sentinel lymph node biopsy in melanoma patients with clinically negative regional lymph nodes-one institution's experience. Melanoma Res 2003;13: $35-43$.

19 de Wilt JH, Thompson JF, Uren RF, et al. Correlation between preoperative lymphoscintigraphy and metastatic nodal disease sites in 362 patients with cutaneous melanomas of the head and neck. Ann Surg 2004;239:544-552.

20 Thompson JF. Sentinel node biopsy. J Surg Oncol 1997;66:270-272.
21 Thompson JF, Niewind P, Uren RF, et al. Single-dose isotope injection for both preoperative lymphoscintigraphy and intraoperative sentinel lymph node identification in melanoma patients. Melanoma Res 1997;7:500-506.

22 Cochran AJ. Surgical pathology remains pivotal in the evaluation of 'sentinel' lymph nodes. Am J Surg Pathol 1999;23:1169-1172.

23 Essner R, Conforti A, Kelley MC, et al. Efficacy of lymphatic mapping, sentinel lymphadenectomy, and selective complete lymph node dissection as a therapeutic procedure for early-stage melanoma. Ann Surg Oncol 1999;6:442-449.

24 Cochran AJ. The pathologist's role in sentinel lymph node evaluation. Semin Nucl Med 2000;30:11-17.

25 Cochran AJ, Essner R, Rose DM, et al. Principles of sentinel lymph node identification: background and clinical implications. Langenbecks Arch Surg 2000;385:252-260

26 Haigh PI, Lucci A, Turner RR, et al. Carbon dye histologically confirms the identity of sentinel lymph nodes in cutaneous melanoma. Cancer 2001;92: 535-541.

27 Dawson M, Doble P, Beavis A, et al. Antimony by ICPMS as a marker for sentinel lymph nodes in melanoma patients. Analyst 2003;128:217-219.

28 Uren RF, Howman-Giles R, Thompson JF, et al. Lymphoscintigraphy to identify sentinel lymph nodes in patients with melanoma. Melanoma Res 1994;4: 395-399.

29 Diaz LK, Hunt K, Ames F, et al. Histologic localization of sentinel lymph node metastases in breast cancer. Am J Surg Pathol 2003;27:385-389.

30 Scolyer RA, Thompson JF, Li LX, et al. Failure to remove true sentinel nodes can cause failure of the sentinel node biopsy technique: evidence from antimony concentrations in false-negative sentinel nodes from melanoma patients. Ann Surg Oncol 2004;11: 174S-178S.

31 Lean CL, Bourne R, Thompson JF, et al. Rapid detection of metastatic melanoma in lymph nodes using proton magnetic resonance spectroscopy of fine needle aspiration biopsy specimens. Melanoma Res 2003;13:259-261. 